\title{
Additive Effekte kombinierter Psycho- und Pharmako- therapie für depressive Patienten: Illusion oder Tatsache?
}

\author{
A. Plattner H.-J. Möller U. Hegerl \\ Psychiatrische Klinik der Ludwig-Maximilians-Universität München
}

\author{
Schlüsselwörter \\ Kombinierte Psychotherapie mit Pharmakotherapie . \\ Review · Chronische Depression · Schwere Depression . \\ Ältere Patienten
}

\section{Zusammenfassung}

Dieser Artikel beschäftigt sich mit der qualitativen Analyse von aktuellen Studien aus den 1990er Jahren zur vergleichenden Wirksamkeit von Psycho- und Pharmakotherapie gegenüber einer kombinierten Therapie. Die Ergebnislage unterstützt insgesamt das Fazit der US-amerikanischen Gesundheitsbehörde, dass der kombinierte Einsatz von Psychotherapie und Pharmakotherapie aufgrund der Datenlage nicht generell für jeden Patienten empfohlen werden kann. In der Akutbehandlung von $\mathrm{Pa}-$ tienten mit "Major Depressive Disorder» (MDD) ist eine Überlegenheit der Kombinationsbehandlung gegenüber Psychotherapie und Pharmakotherapie alleine nicht nachweisbar. Bezogen auf die Langzeitbehandlung von MDD-Patienten zeigt die methodisch herausragende Studie aus der Arbeitsgruppe um Frank, dass eine Kombinationsbehandlung der Behandlung mit nur interpersoneller Psychotherapie überlegen ist, nicht aber der Behandlung mit Trizyklika. Dieses Bild differenziert sich weiter, wenn man Subgruppen von Patienten betrachtet: Patienten mit schwereren Formen chronischer Depressionen und ältere Patienten profitieren vor allem in Bezug auf die Langzeitbehandlung. Ergebnisse einer Mega-Analyse belegen, dass bei mittleren bis schweren depressiven Episoden einer MDD eine Akutbehandlung mit kombinierter Pharmako-Psychotherapie zu einer deutlicheren und bedeutsam schnelleren Symptomreduktion führt als Psychotherapie allein.

\author{
Key Words \\ Combined psychotherapy and pharmacotherapy . \\ Review · Chronic depression - Severe depression . \\ Elderly patients
}

\section{Summary}

Additive Effects of Combined Psycho- and Pharmacotherapy for Depressed Patients: Illusion or Fact?

This article qualitatively reviews the comparative efficacy of pharmacotherapy and psychotherapy vs either modality alone. Recent results are confirming AHCPR statements, that, on the basis of current data, in acute treatment of Major Depressive Disorders (MDD), the routine use of both psychotherapy and pharmacotherapy is not warranted. But a methodologically outstanding study from the work-group around Frank shows that, in longterm treatment of MDD-patients, combined psycho-pharmaocotherapy is superior compared to interpersonal psychotherapy alone, but not compared to tricyclics alone. In two settings patients might benefit substantially from a combination treatment compared to both psychotherapy and pharmacotherapy alone: 1) Acute and long-term treatment of more severe chronic depression, and 2) long-term treatment of elderly MDD-patients. More severely depressed MDD-patients profit more and faster if treated with combined psycho-pharmacotherapy compared compared to psychotherapy alone.

\begin{tabular}{ll}
\hline KARGER & ( ) 2001 S. Karger GmbH, Freiburg \\
$\begin{array}{l}\text { Fax +49761 4520714 } \\
\begin{array}{l}\text { E-mail Information@Karger.de } \\
\text { www.karger.com }\end{array}\end{array}$ & Accessible online at: \\
www.karger.com/journals/ver
\end{tabular}

Dr. rer. biol. hum. Dipl.-Psych. A. Plattner Kompetenznetz «Depression», Klinik für Psychiatrie und Psychotherapie der Ludwig-Maximilians-Universität München

Nußbaumstraße 7, D-80336 München (Deutschland)

Tel. +49 89 5160-5556 (oder -5541), Fax -5542

E-mail Anita.Plattner@psy.med.uni-muenchen.de 


\section{Einleitung}

Die Kombination von Psychotherapie und Pharmakotherapie ist eine weit verbreitete Behandlung für Depressionen, obwohl die vorliegenden Studienergebnisse nach wie vor kontrovers diskutiert werden. Unter anderem wird eine solche Parallelbehandlung von der American Psychiatric Association generell empfohlen [APA, 1993]. Bedenken wegen der unsicheren Beweislage äußerte im Gegensatz dazu die Agency for Health Care Policy and Research (AHCPR) in ihren Richtlinien zur Behandlung von Depressionen in der Primärversorgung [Depression Guideline Panel, 1993].

Obwohl bereits sowohl qualitative [systematisch zuletzt von Hollon et al., 1991] als auch quantitative Literaturanalysen [zuletzt Gaffan et al., 1995] zu diesem Thema vorliegen, sind gerade die dort nicht eingeschlossenen Studien, die seit Anfang oder Mitte der 1990er Jahre veröffentlicht wurden, von besonderem Interesse, weil Sie größere Stichproben analysieren. Die Gefahr eines Publikationsbias ist in diesem Fall generell geringer, auch wenn die Verzerrung der Ergebnisse durch subjektive Forschungspräferenzen («Researcher Allegiance» [Gaffan et al., 1995]) bei Therapievergleichsstudien nicht generell ausgeschlossen werden kann. Die jüngeren Studien erfüllen insgesamt eher die Kriterien einer evidenzbasierten Forschung [Sackett, 1998] und sollen daher, neben einer kurzen Zusammenfassung der Ergebnisse aus älteren qualitativen und quantitativen Analysen, den Schwerpunkt der vorliegenden Übersichtsarbeit bilden.

Einschlusskriterien für in diese qualitative Analyse eingeschlossenen Studien sind: Publiziert nach 1990, Originalpublikation einer randomisierten Studie, Zellgrößen von mindestens 20 und Blindheit der «Rater» gegenüber der Art der Behandlung. Studien zur Langzeitbehandlung mussten eine initiale Akutbehandlungsphase enthalten und die aktiven Behandlungen mussten während der Weiterbehandlungs- bzw. Erhaltungstherapie fortgesetzt werden. Unter Bezug auf die Definitionen der Amerikanischen Gesundheitsbehörde [Depression Guideline Panel, 1993] wird unter Weiterbehandlung die Verhütung eines Rückfalls (d.h. die Rückkehr der gegenwärtigen depressiven Episode) verstanden. Eine Weiterbehandlung dauert 4-9 Monate, nachdem der Patient ein Ansprechen auf die Behandlung gezeigt hat. Eine anschließende Erhaltungstherapie kann mehrere Jahre dauern und soll das Auftreten einer neuen Episode verhindern. Abbildung 1 zeigt den möglichen Verlauf einer Depression in den verschiedenen Behandlungsphasen.

Die folgende Analyse der Studien ist in zwei Hauptteile untergliedert, wobei Studien zur Akut- und zur Langzeitbehandlung jeweils separat dargestellt werden:

- Der erste Teil beschäftigt sich mit der generellen Beweislage für additive Effekte einer kombinierten Psychopharmakotherapie im Vergleich zu Einzelbehandlungen. Es folgt ein kurzer Überblick über die Ergebnisse von Meta-Analysen und über ältere Therapievergleichstudien die bereits in qua-

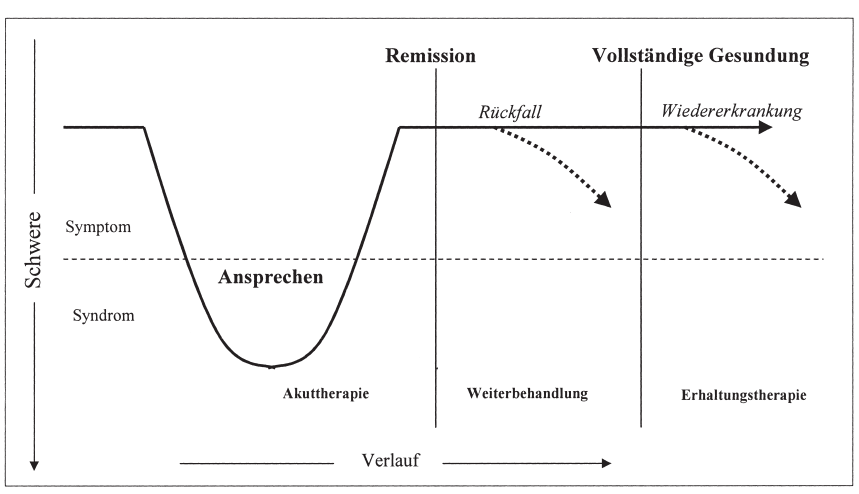

Abb. 1. Fünf mögliche Wendepunkte während einer Behandlung: Ansprechen, Remission, Rückfall, Gesundung, Wiedererkrankung (Rezidiv). Adaptiert aus Kupfer [1991].

litativen Reviews dargestellt worden sind. Die älteren Studien beschäftigen sich vor allem mit der Behandlung akuter Episoden einer Major Depressive Disorder (MDD) ohne die Schwere oder den Verlauf der Depression weiter zu charakterisieren.

- Im zweiten Teil werden die neueren Studien aus den 1990er Jahren im Detail diskutiert. Zunächst geht es dabei um die Replikation der älteren Studien, wie sie oben beschrieben worden sind. Den Schwerpunkt des zweiten Teils bilden Studien die an Subgruppen von depressiven Patienten durchgeführt worden sind, wobei vor allem die Frage beleuchtet wird, ob die Schwere oder der Verlauf einer Depression relevant sind für die Wahl einer kombinierten Behandlung gegenüber einer Einzelbehandlung.

\section{Allgemeine Hinweise für additive Effekte auf der Basis von Meta-Analysen und älteren Studien}

In ihrem Review der älteren Studien, die bis 1990 publiziert worden waren, schließen Hollon und seine Mitarbeiter [Hollon et al., 1991], dass es in einigen Studien zwar Trends oder Tendenzen [Blackburn et al., 1981; Murphy et al., 1984; Covi und Lipman, 1987], aber keine statistisch signifikanten Ergebnisse gibt, die für eine Überlegenheit einer kombinierten Behandlung gegenüber einer Einzelbehandlung sprechen. Diese Schlussfolgerung bezieht sich auf eine Reihe von Therapievergleichsstudien zur Behandlung akuter Episoden einer MDD. Auch drei Meta-Analysen von Studien aus dem gleichen Publikationszeitraum ergeben keine signifikanten Erfolgsunterschiede zwischen einer kombinierten Therapie und einer Einzeltherapie. In Tabelle 1 sind die Kennwerte und Ergebnisse dieser Meta-Analysen wiedergegeben: Die aktuellste stammt von Gaffan et al. [1995] und die aktuellste Studie, die in diese Analyse eingeschlossen worden war, stammt von 1987. Die mittlere Zellgröße der 5-11 eingeschlossenen Studien liegt bei ungefähr 14. Die Art und Qualität der Medikation wird nicht immer berichtet. 
Tab. 1. Meta-Analysen zur Akutbehandlung: Vergleich zwischen Kombinierter Pharmako-Psychotherapie und Monotherapie

\begin{tabular}{|c|c|c|c|c|c|c|}
\hline \multirow[t]{2}{*}{ Autor } & \multirow{2}{*}{$\begin{array}{l}\text { Publikations- } \\
\text { zeitraum }\end{array}$} & \multirow{2}{*}{$\begin{array}{l}\text { Mittlere Zellgröße } \\
\text { (range) }\end{array}$} & \multirow[t]{2}{*}{ Pharm } & \multirow[t]{2}{*}{ Erfolgskriterien } & \multicolumn{2}{|l|}{ Ergebnisse } \\
\hline & & & & & Kombi $>$ PT & Kombi $>$ M \\
\hline Conte et al. [1986] & 1974-1984 & - & meist AMI & verschieden & $(\mathrm{JA})^{*}$ & $(\mathrm{JA})^{*}$ \\
\hline Gaffan et al. [1995] & 1976-1987 & $14,6(2-39)$ & - & BDI & NEIN & - \\
\hline Robinson et al. [1990] & 1976-1986 & $14,3(4-47)$ & verschieden & gepoolt $^{1}$ & NEIN & NEIN \\
\hline
\end{tabular}

NEIN = kein Unterschied; (JA) = leichte Überlegenheit (kein Signifikanztest); - = nicht analysiert; BDI = Beck Depressions Inventar; AMI = Amitriptylin; Kombi = kombinierte Psycho-Pharmakotherapie; PT = Psychotherapie; $\mathrm{M}=\mathrm{Medikation}$;

${ }^{1}$ BDI, Zung Selbst-Rating Depressions-Skala, Hamilton-Rating-Scale for Depression (HRSD) usw.

Weder die Gaffan- noch die Robinson-Meta-Analyse [Gaffan et al., 1995; Robinson et al., 1990] fand einen Unterschied zwischen kombinierter Psycho-Pharmakotherapie und jeder Behandlungsbedingung für sich allein. Nur Conte [Conte et al., 1986], der die größte Anzahl von 11 Studien analysiert hat, berichtet über die leichte Überlegenheit einer Kombinationsbehandlung im Vergleich zu Psychotherapie als auch zu Pharmakotherapie. Allerdings sind die Ergebnisse von Conte schwer mit denjenigen aus den anderen Meta-Analysen zu vergleichen, weil er eine ungewöhnliche qualitativ-quantiative statistische Technik verwendet hat. Außerdem ist anzumerken, dass die Conte-Analyse nur die sehr alten Studien eingeschlossen hat, die zwischen 1974 und 1984 publiziert worden sind. Diese Studien weisen sehr kleine Zellgrößen auf und haben daher ein erhöhtes Risiko ein «Publikation-Bias» aufzuweisen. Gaffan [Gaffan et al., 1995] konnte außerdem nachweisen, dass diese älteren Studien stärker von subjektiven Forschungsinteressen beeinflusst waren.

\section{Studien aus den 1990er Jahren}

Wie aus dem vorhergehenden Abschnitt zu ersehen ist, sind die Studien aus den 1990er Jahren bis heute nicht systematisch reviewt worden. Die bisher qualitativ analysierten Studien waren vor 1990 publiziert worden und wie Tabelle $1 \mathrm{zu}$ entnehmen ist, basieren die quantitativen Analysen auf Studien aus den Jahren 1976 bis 1987 . Kennwerte der in die vorliegende Arbeit eingeschlossenen Studien aus den 1990er Jahren (Stichprobenkennwerte, Studienarme, Art und Dosierung der Medikation, Dauer und Häufigkeit der Psychotherapie und Haupterfolgskriterien) und ihre Ergebnisse sind in Tabelle 2 wiedergegeben.

Ein erster Blick auf Tabelle 2 ergibt das folgende Bild: 8 Studien wurden in den 1990er Jahren insgesamt durchgeführt. 5 davon beziehen sich auf Akutbehandlung und 3 auf Langzeitbehandlung. Die meisten Studien weisen 3 Behandlungsarme auf, für gewöhnlich Psychotherapie, Pharmakotherapie und deren Kombination. 2 Studien berichten über zusätzliche Plazebo-Kontrolle und die Studie von Frank et al. [1990] verfügt sogar über einen Kontrollarm für die kombinierte Behandlungsbedingung bestehend aus Plazebo plus klinisches Management. Die mittlere Stichprobengröße ist 140 (range: 107-191) und die mittlere Zellgröße ist 46,4 (range: 35,7-80,9) - wenn man die Keller-Studie [Keller et al., 2000] mit einer Stichprobengröße von 681 als Ausreißer betrachtet und nicht berücksichtigt. Die klare Mehrheit der Patienten wurde ambulant mit Trizyklika behandelt. Der Median der Psychotherapiedauer liegt bei 12 Wochen in den Akutbehandlungsstudien. Das Haupterfolgskriterium für den Therapieerfolg ist die «Hamilton Rating Scale for Depression» (HRSD). Die Ergebnisse sind in beiden Richtungen dargestellt: kombinierte Therapie versus Psychotherapie und kombinierte Therapie versus Medikation.

Wenn man die Studienkennwerte und Ergebnisse aus den Tabellen 1 und 2 vergleicht, dann fällt auf, dass in den älteren Studien die Zellgrößen substantiell geringer ausfallen und daher die Wahrscheinlichkeit signifikante Ergebnisse zu erhalten gering bleiben dürfte. Ein anderer Unterschied betrifft die Tatsache, dass die älteren Studien offensichtlich verschiedene Haupterfolgkriterien verwendet haben und in den neueren Studien einheitlich die HRSD zur Anwendung gekommen ist. Nicht zuletzt zeigen die Ergebnisse aus Tabelle 2 ein vielversprechenderes Bild zugunsten der Überlegenheit einer Kombinationsbehandlung über sowohl Psychotherapie als auch Pharmakotherapie allein. Im Folgenden werden die Studien aus Tabelle $2 \mathrm{im}$ Detail beschrieben:

\section{Major Depressive Disorder}

Auch die überwiegende Mehrheit der Studien aus den 1990er Jahren konzentriert sich auf die Behandlung einer akuten MDD ohne bei den Einschlusskriterien genauer auf den Schweregrad oder den Verlauf der Depression einzugehen. Es gibt aus dieser Zeit 3 Studien zur Akutbehandlung und 2 Studien zur Erhaltungstherapie.

Akutbehandlung: Bezogen auf die Akutbehandlung einer MDD sind die nicht-signifikanten Ergebnisse der älteren Studien in 3 neueren und größeren Studien bestätigt worden 
Tab. 2. Studien (1990-2000): Vergleich zwischen Kombinierter Pharmako-Psychotherapie und Monotherapie

\begin{tabular}{|c|c|c|c|c|c|c|c|}
\hline \multirow[t]{2}{*}{ Autor } & \multirow[t]{2}{*}{ Beispiel $^{1}$} & \multirow[t]{2}{*}{ Gruppen } & \multirow{2}{*}{$\begin{array}{l}\text { Medikation } \\
\text { (Dosierung, mg/Tag) }\end{array}$} & \multirow{2}{*}{$\begin{array}{l}\text { Dauer/n } \\
\text { Sitzungen }\end{array}$} & \multirow{2}{*}{$\begin{array}{l}\text { Haupterfolgs- } \\
\text { kriterien }\end{array}$} & \multicolumn{2}{|l|}{ Ergebnisse } \\
\hline & & & & & & Kombi $>$ PT & Kombi $>$ M \\
\hline \multicolumn{8}{|c|}{ Akutbehandlung einer MDD (keine Spezifikation der Einschlusskriterien hinsichtlich Schwere oder Verlauf) } \\
\hline $\begin{array}{l}\text { Hollon et al. } \\
{[1992]}\end{array}$ & 107 amb. Pat. & $\begin{array}{l}\text { M } \\
\text { KT } \\
\text { M+KT }\end{array}$ & $\begin{array}{l}\text { Imipramin } \\
\text { (verschieden) }\end{array}$ & $12 \mathrm{~W} / \max .20$ & HRSD, GAS & NEIN & NEIN \\
\hline $\begin{array}{l}\text { Mynors-Wallis et al. } \\
\text { [2000] }\end{array}$ & 151 Hausarztpat. & $\begin{array}{l}\text { PL-HA } \\
\text { PL-PF } \\
\text { M } \\
\text { PL+M }\end{array}$ & $\begin{array}{l}\text { Fluvoxamin, Paroxetin } \\
\text { (verschieden) }\end{array}$ & $12 \mathrm{~W} / 6$ & HRSD, BDI & NEIN & NEIN \\
\hline $\begin{array}{l}\text { Hautzinger et al. } \\
{[1996]}\end{array}$ & $\begin{array}{l}75 \text { stat. Pat., } 116 \text { amb. } \\
\text { Pat. } \\
\text { nicht endogene } \\
\text { Depression }\end{array}$ & $\begin{array}{l}\mathrm{M} \\
\mathrm{KT} \\
\mathrm{M}+\mathrm{KT}\end{array}$ & $\begin{array}{l}\text { Amitriptylin } \\
\text { (verschieden) }\end{array}$ & $8 \mathrm{~W} / 24$ & HRSD, BDI & NEIN & NEIN \\
\hline \multicolumn{8}{|c|}{ Langzeitbehandlung einer MDD (keine Spezifikation der Einschlusskriterien hinsichtlich Schwere oder Verlauf) } \\
\hline $\begin{array}{l}\text { Frank et al. } \\
{[1990]}\end{array}$ & $\begin{array}{l}128 \text { Pat. mit } \\
\text { rezidivierender } \\
\text { Depression }\end{array}$ & $\begin{array}{l}\mathrm{M}+\mathrm{KM} \\
\mathrm{PL}+\mathrm{KM} \\
\mathrm{IPT} \\
\mathrm{IPT}+\mathrm{PL} \\
\mathrm{IPT}+\mathrm{M}\end{array}$ & $\begin{array}{l}\text { Imipramin } \\
(150-300)\end{array}$ & $\begin{array}{l}3 \mathrm{~J} / \\
\text { 1Sitzung pro } \mathrm{M}\end{array}$ & HRSD & JA & NEIN \\
\hline $\begin{array}{l}\text { Reynolds et al. } \\
\text { [1999] }\end{array}$ & $\begin{array}{l}107 \text { Pat. mit } \\
\text { rezidivierender } \\
\text { Depression }\end{array}$ & $\begin{array}{l}\mathrm{M}+\mathrm{KM} \\
\mathrm{KM}+\mathrm{PL} \\
\mathrm{IPT}+\mathrm{M} \\
\mathrm{IPT}+\mathrm{PL}\end{array}$ & $\begin{array}{l}\text { Nortriptylin } \\
(80-120 \mathrm{ng} / \mathrm{ml})\end{array}$ & $\begin{array}{l}3 \mathrm{~J} / \\
\text { 1Sitzung pro } \mathrm{M}\end{array}$ & HRSD & JA & $(\mathrm{JA})$ \\
\hline $\begin{array}{l}\text { Akute Behandlung ei } \\
\text { deJong-Meyer et al. } \\
\text { [1996] }\end{array}$ & $\begin{array}{l}\text { er schweren MDD } \\
155 \text { stat. + amb. Pat. }\end{array}$ & $\begin{array}{l}\mathrm{M}+\mathrm{UT} \\
\mathrm{M}+\mathrm{KT}\end{array}$ & $\begin{array}{l}\text { Amitriptylin } \\
\text { (verschieden) }\end{array}$ & $8 \mathrm{~W} / 24$ & HRSD, BDI & NEIN & $\begin{array}{l}\text { Ja/amb. Pat. } \\
\text { Nein/stat. Pat. }\end{array}$ \\
\hline \multicolumn{8}{|c|}{ Akute Behandlung einer chronischen Depression } \\
\hline $\begin{array}{l}\text { Keller et al. } \\
{[2000]}\end{array}$ & $\begin{array}{l}681 \text { Pat. mit } \\
\text { chronischer } \\
\text { Depression }\end{array}$ & $\begin{array}{l}\mathrm{M} \\
\mathrm{PT}^{2} \\
\mathrm{M}+\mathrm{PT}\end{array}$ & $\begin{array}{l}\text { Nefazodon } \\
(300-600)\end{array}$ & $12 \mathrm{~W}$ & HRSD-24 & JA & JA \\
\hline $\begin{array}{l}\text { Langzeitbehandlung } \\
\text { Paykel et al. } \\
\text { [1999] }\end{array}$ & $\begin{array}{l}\text { ner chronischen Depress } \\
158 \text { Pat. mit } \\
\text { residualer Depression }\end{array}$ & $\begin{array}{l}\mathrm{M}+\mathrm{KM} \\
\mathrm{M}+\mathrm{KM}+\mathrm{K}\end{array}$ & $\begin{array}{c}\text { verschieden } \\
\mathrm{T}(\text { verschieden })\end{array}$ & $17 \mathrm{M} / 20+2$ & HRSD-17 & - & JA \\
\hline $\begin{array}{l}1=\text { falls keine weitere } \\
2=\text { das kognitive Verl } \\
\text { Psychotherapie verw } \\
\text { JA = signifikant über } \\
\text { amb. = ambulante; K } \\
\text { durchgeführt von Pfl } \\
\text { PT = Psychotherapie; } \\
\text { Depression; GAS = C }\end{array}$ & $\begin{array}{l}\text { Spezifikationen angege } \\
\text { altensanalysesystem kom } \\
\text { adet werden. } \\
\text { gen; }(\mathrm{JA})=\text { Trend zur Ü } \\
\text { I = Klinisches Managem } \\
\text { gepersonal; M = Medika } \\
\text { PL = Plazebo; W = Woch } \\
\text { lobal Assessment Scale; }\end{array}$ & $\begin{array}{l}\text { n sind, müs } \\
\text { niert mehre } \\
\text { rlegenheit; } \\
; \text { PL-HA = } \\
\text {; KT = ko } \\
\text { M = Mona } \\
\mathrm{I}=\text { Beck D }\end{array}$ & $\begin{array}{l}\text { en die Patienten die Diag } \\
\text { re behaviorale, kognitive } \\
\text { NEIN = keine Überlegen } \\
\text { Problemlösungsbehandlu } \\
\text { nitive Therapie; UT = un } \\
\text {; = Jahr; max. = maxima } \\
\text { epressions Inventar. }\end{array}$ & $\begin{array}{l}\text { gnosekriterien fü } \\
\text { und interperson } \\
\text { heit; - = nicht an } \\
\text { ang durchgeführt } \\
\text { terstützende The } \\
\text { le Zahl der Sitzu }\end{array}$ & $\begin{array}{l}\text { r eine akute Epi } \\
\text { elle Techniken, } \\
\text { alysiert; Pat. = P } \\
\text { von Hausärzten } \\
\text { rapie; IPT = int } \\
\text { ngen; HRSD = I }\end{array}$ & $\begin{array}{l}\text { ode einer MD } \\
\text { le in anderen F } \\
\text { atienten; stat. } \\
\text { PL-PF = Prob } \\
\text { rpersonelle Ps } \\
\text { Iamilton Ratin }\end{array}$ & $\begin{array}{l}\text { erfüllen; } \\
\text { ormen der } \\
\text { stationäre; } \\
\text { emlösung } \\
\text { chotherapie; } \\
\text {-Skala für }\end{array}$ \\
\hline
\end{tabular}

[Hollon et al., 1992; Hautzinger et al., 1996; Mynors-Wallis et al., 2000]:

- Hollon und seine Mitarbeiter [1992] verglichen die Wirksamkeit von kognitiver Psychotherapie und Imipramin jeweils einzeln und in Kombination. 107 Patienten wurden randomisiert einer 12-wöchigen Behandlung zugewiesen. Die Ergebnisse der Studie erbrachten für jede Patientengruppe signifikante Symptomreduktionen, aber keinen sig- nifikanten Vorteil der kombinierten Behandlung gegenüber einer Monotherapie und auch keine Trends in diese Richtung.

- Eine andere große Studie aus dem deutschen Sprachraum [Hautzinger et al., 1996] erforschte die Wirksamkeit von Amitryptilin plus klinisches Management, kognitive Verhaltentherapie alleine, und einer Kombination aus beiden Behandlungen. 191 stationäre und ambulanten Patienten wur- 
den allen drei Behandlungsbedingungen zugewiesen. In beiden Settings waren alle drei Behandlungen gleich effizient in der Symptomreduktion sowohl auf der HRSD als auch auf dem Beck-Depressions-Inventar (BDI), aber keine Behandlung war der anderen überlegen. Patienten, die eine Kombinationsbehandlung erhalten hatten, zeigten zwar höhere Response-Raten (Response-Kriterien: BDI < 10 und HRSD $<10$ ), aber es gab keine statistisch signifikanten Unterschiede oder Trends.

- Vor kurzem führte Mynors-Wallis et al. [2000] eine Studie an 151 Patienten durch. Die im Hausarztbereich rekrutierten Patienten wurden entweder einer 12-wöchigen Problemlösetherapie, kombiniert mit Medikation, zugewiesen oder den Behandlungsbedingungen jeweils alleine. Auch hier zeigten alle Patienten eine klare Verbesserung über die 12wöchige Behandlung, aber die Kombination war ebenfalls nicht effektiver als jede Behandlungsbedingung für sich allein.

Insgesamt ist aus den Ergebnissen der Studien nicht ableitbar, dass ambulante Patienten, die an einer akuten Episode einer MDD leiden, generell mit einer Kombination von Psychotherapie und Pharmakotherapie behandelt werden müssen. In den jüngeren Studien die in diesem Abschnitt beschrieben worden sind, zeigten sich auch keine statistischen Trends wie sie aus den älteren Studien berichtet worden waren.

Langzeitbehandlung: Aus der Arbeitsgruppe um Kupfer gibt es zwei Studien zur Erhaltungstherapie für MDD-Patienten. Bezogen auf die Methode und das Design führten Frank und ihre Mitarbeiter [Frank et al., 1990] die derzeit wohl fundierteste Studie zu der hier interessierenden Fragestellung durch. In einem 5-armigen Design verglichen sie den Prozentsatz des Wiederauftretens einer Depression über einen Zeitraum von 3 Jahren hinweg. Die Patienten erhielten entweder klinisches Management und Imipramin, klinisches Management und Plazebo, interpersonelle Psychotherapie (IPT) allein, IPT und Plazebo oder IPT und Imipramin. Die Patienten wurden nach einer 2-wöchigen medikationsfreien «Washout-Phase» diesen Behandlungsbedingungen zufällig zugewiesen. Alle Messinstrumenterhebungen wurden vor und nach dieser initialen Phase erhoben. Während einer 12-wöchigen Akutbehandlung wurde die IPT wöchentlich durchgeführt, in der Weiterbehandlung wurde sie 2-wöchentlich durchgeführt und in der Erhaltungstherapie erfolgte die IPT dann monatlich. Imipramin wurde über die gesamte Behandlungsdauer von 3 Jahren auf der Dosis beibehalten, die schon in der Akutbehandlungsphase verabreicht worden war. Die Autoren fanden eine signifikante Überlegenheit der Kombination von Imipramin plus IPT gegenüber IPT alleine, nicht aber gegenüber der Behandlung mit Pharmakotherapie allein.

In einer ähnlichen Studie, die mit älteren Patienten mit einer teilremittierten MDD durchgeführt worden ist [Reynolds et al., 1999], wurde die Wirksamkeit von Nortriptylin und IPT einzeln und in Kombination zur Prävention des Wiederauftretens einer MMD-Episode untersucht. Von insgesamt 187 in die Studie eingeschlossene Patienten waren 107 nach einer offenen Akut- und Erhaltungstherapie mit Nortriptylin und IPT vollständig gesundet. Diese Patienten wurden randomisiert einer anschließenden 3-jährigen Langzeittherapie mit 4 Therapiebedingungen zugewiesen: Monatliches Klinisches Management mit Nortriptylin-Hydrochlorid, klinisches Management mit Plazebo, monatliche Langzeittherapie mit IPT plus Plazebo, und monatliche Langzeittherapie mit IPT plus Nortriptylin. Die Ergebnisse zeigten, dass die kombinierte Behandlung mit Nortriptylin plus IPT der Kombination von IPT und Plazebo signifikant überlegen war, und es ergab sich auch eine fast signifikante Überlegenheit der kombinierten Therapie gegenüber Nortriptylin Monotherapie $(p=0,06)$.

Diese Ergebnisse zeigen insgesamt, dass in der Langzeitbehandlung einer Major Depression eine Kombinationsbehandlung zwar einer interpersonellen Psychotherapie überlegen ist, nicht aber einer Behandlung mit Trizyklika, wenn die Dosis der Akutbehandlung beibehalten wird. Allerdings gibt die Studie von Reynolds einen ersten Hinweis, dass es bei der Langzeitbehandlung älterer Patienten einen additiven Effekt der kombinierten Psycho-Pharmakotherapie nicht nur gegenüber Psychotherapie, sondern auch gegenüber einer Behandlung mit Antidepressiva gibt. Wie man einer abermaligen Betrachtung von Tabelle 2 entnehmen kann, sind die Schwere und der Verlauf einer depressiven Störung andere vielversprechende Kriterien um Behandlungsvorteile einer kombinierten Therapie gegenüber Monotherapie nachweisen zu können.

\section{Schwere Ausprägungen der Major Depression}

Bei schweren Ausprägungen einer MDD liegen zur vergleichenden Wirksamkeit von kombinierter Psychotherapie-Pharmakotherapie gegenüber Monotherapie 2 Studien zur Akutbehandlung [Thase et al., 1997; deJong-Meyer et al., 1996] und eine Studie zur Langzeitbehandlung vor. Beide Studien lehnten sich bei der Definition schwerer Depressionen an die bekannte Studie von Elkin [Elkin et al., 1989] an, in der schwereren Depressionen ein HRSD-Wert von $>20$, und milderen Depressionen ein HRSD-Wert von $\leq 19$ zugeordnet worden war.

In einer 3-zentrischen Studie von deJong-Meyer und ihren Mitarbeitern [1996], die an stationären und ambulanten Patienten mit endogener Depression durchgeführt worden war, wurden 155 Patienten zufällig entweder einer Kombinationsbehandlung aus kognitiv-behavioraler Therapie und Amitriptylin oder der Bedingung Amitriptylin plus unterstützende Gespräche zugewiesen. Depressions-Scores (BDI und HRSD $>20$ ) charakterisierten die Stichprobe als schwer depressiv mit einer langen Vorgeschichte depressiver Störungsbilder und deren Behandlung. Haupterfolgskriterien bei Post-Erhebung waren der BDI und die HRSD, letztere wurde von unabhängigen, blinden klinischen Ratern durchgeführt. Alle Pa- 
tienten zeigten sehr signifikante Reduktionen der depressiven Symptomatik, aber die stationären Patienten verbesserten sich noch einmal signifikant mehr als die ambulanten Patienten. Varianzanalysen bei Post-Erhebung ergaben keine Unterschiede zwischen den beiden Behandlungsbedingungen. Sekundäranalysen zeigten, dass für stationäre Patienten die Verbesserung der Symptome und die Responder-Raten hinsichtlich der verschiedenen Behandlungsbedingungen vergleichbar waren, für ambulante Patienten ergab sich aber ein signifikanter Vorteil für die kombinierte Therapie.

In ihrer Mega-Analyse (Meta-Analyse mit Rohdaten) von 6 randomisierten klinischen Studien konnten Thase und seine Kollegen [1997] zeigen, dass bei schwereren Ausprägungen einer MDD (HRSD > 20) eine Kombination von kognitiver Verhaltenstherapie und Trizyklika zu signifikant höheren Gesundungsraten führte als Psychotherapie allein. Gesundung wurde dabei als 4-wöchige Zeitspanne mit einem HRSDScore von weniger als 7 definiert, der bis Woche 16 beibehalten werden konnte. Survival-Analysen zeigten außerdem, dass eine Kombinationsbehandlung die Symptome einer schweren Depression schneller reduzieren konnte als das die Psychotherapie allein vermochte. Patienten die zusätzlich zur Psychotherapie Pharmakotherapie erhalten hatten, zeigten signifikant schnellere Gesundungsverläufe als solche Patienten die nur mit Psychotherapie behandelt worden waren. Sowohl bei den Gesundungsraten als auch bei den Survival-Analysen ergaben sich keine signifikanten Gruppenunterschiede für Patienten mit milderen Ausprägungen einer MDD, d.h. mit einem initialen HRSD-Score von $\leq 19$. Leider ist in dieser Analyse kein Vergleich der kombinierten Therapie gegenüber der Pharmakotherapie allein eingeschlossen worden, so dass die Aussage nur auf das Verhältnis von kombinierter Therapie gegenüber Psychotherapie begrenzt werden kann und keine Aussage über das Verhältnis der Kombinationsbehandlung gegenüber der Pharmakotherapie möglich ist.

\section{Major Depression mit chronischem Verlauf}

Im Gegensatz zu den Ergebnissen bei schweren Ausprägungen einer MDD sind die Ergebnisse für an einer MDD mit chronischem Verlauf leidenden Patienten deutlich: Eine kombinierte Psycho-Pharmakotherapie ist der Monotherapie eindeutig überlegen. Eine MDD mit chronischem Verlauf kann folgende Diagnosen betreffen: Chronische MDD, Rezidivierende MDD mit unvollständiger Gesundung zwischen den Episoden (z.B. Minore Depression) und doppelte Depression (Dysthymie kombiniert mit MMD). Aus den 1990er Jahren gibt es jeweils eine Studie zur Akutbehandlung [Keller et al., 2000] und zur Erhaltungstherapie [Paykel et al., 1999].

Akutbehandlung: In einer 12-zentrischen Studie mit chronisch depressiven Patienten [Keller et al., 2000] wurde die Kombination von Nefazodon und kognitiver Verhaltenstherapie gegenüber der Wirksamkeit jeder Behandlungsbedingung für sich alleine untersucht. 681 ambulante Patienten wurden randomisiert einer 12-wöchigen Behandlung mit Nefazodon, Psychotherapie (16-20 Sitzungen) oder beidem zugewiesen. Die Patienten mussten eine Diagnose einer chronischen Depression aufweisen, in diesem Fall eine Episode einer MDD von mindestens 2 Jahren Dauer oder eine doppelten Depression. Als Psychotherapie wurde das sogenannte «kognitiv-behaviorale Analysesystem» verwendet. Diese neue Technik eine Kombination aus kognitiv-behavioralen und interpersonellen Methoden ist speziell zur Behandlung chronischer Formen entwickelt worden. Eine Remission d.h. eine vollständige Symptomreduktion wurde definiert als ein Score von 8 oder weniger auf der HRSD nach 10 und 12 Wochen. Für Patienten die keine Remission erreichten wurde eine zufriedenstellende Response definiert als eine Reduktion um mindestens 50\% auf der HRSD verglichen mit der Baseline, wobei ein HRSDScore von 15 unterschritten werden musste. Die durchschnittliche Responserate (sowohl Remission als auch zufriedenstellende Response) war $48 \%$ in sowohl der Nefazodon-Gruppe als auch der Psychotherapiegruppe im Vergleich zu 73\% in der kombinierten Behandlungsgruppe.

Erhaltungstherapie: In der schwer zu behandelnden Gruppe von Patienten mit unvollständiger Gesundung zwischen den Episoden einer MDD, die nur zum Teil auf eine Akutbehandlung mit Antidepressiva angesprochen hatten, konnte die kognitive Therapie das Behandlungsergebnis wesentlich verbessern [Paykel et al., 1999]. Paykel und seine Kollegen führten eine große randomisierte Studie durch und verglichen dabei kognitive Therapie plus klinischer Standardbehandlung mit klinischer Standardbehandlung allein. In die Studie eingeschlossen wurden 158 Patienten mit akuter MDD, die nach Antidepressiva-Behandlung teilremittiert waren (mittlere Tagedosen etwa $185 \mathrm{mg}$ Amitriptylin oder $33 \mathrm{mg}$ Fluoxetin), aber die unter einer Residualsymptomatik von 2-18 Monaten Dauer litten. Residuale Symptome mussten mindesten 8 Punkte auf der HRSD und 9 auf dem BDI erreichen. Die Psychotherapie bestand aus 16 Sitzungen während 20 Wochen mit 2 abschließenden Auffrischungssitzungen. Während der 20 Wochen wurden regelmäßig Erfolgsmessungen durchgeführt, zusätzlich auch ein Jahr nach der Behandlung. Die Patienten erhielten antidepressive Behandlung während Erhaltungstherapie und während Langzeittherapie mit der selben Dosierung wie in der Akutbehandlung. Die Rückfallraten nach 68 Wochen waren signifikant reduziert von $47 \%$ in der Gruppe mit der klinischen Standardbehandlung, bis zu 29\% in der Gruppe mit zusätzlicher kognitiver Therapie. Zusätzliche kognitive Therapie erhöhte außerdem die Rate der vollständigen Gesundung, aber dieser Effekt war vergleichsweise klein und zeigte keine signifikanten Effekte in Bezug auf die Symptom-Ratings.

Es gibt also sowohl aus den Akut- als auch von den Langzeitbehandlungsstudien sehr gute Hinweise, dass bei chronischen Verläufen der MDD eine Kombination aus kognitiver Verhaltenstherapie und Medikation einer Monotherapie überlegen ist. 


\section{Methodologische Überlegungen}

Bevor die Ergebnisse der hier dargestellten Studien inhaltlich diskutiert werden sollen, sollten drei wichtige methodologische Probleme erwähnt werden, die für die Interpretation der Ergebnisse wesentlich sind. Beleuchtet werden sollen die Bedeutung von «Dropouts» in Effektivitätsstudien, von Studien mit zu kleinen Zellgrößen und die verschiedenen möglichen Arten von Effekten durch die Kombination zweier verschiedener Behandlungsverfahren.

Dropouts: In der Mehrzahl der Studien wurde ein «intent-totreat» Modell verwendet [deJong-Meyer, 1996; Hautzinger et al., 1996; Keller et al., 2000; Paykel et al., 1999]. Zwei Studien berichten (zusätzlich) separat über die Ergebnisse von Studienbeendern («Completers») und allen einer Behandlungsbedingung zugewiesenen Patienten («all assigned») [Hollon et al., 1991; Paykel et al., 1999]. Von statistischer Seite wird häufig kritisiert, dass Intent-to-treat-Analysen einen Teil der klinisch relevanten Information unterschlagen, z.B. den Grund für das Ausscheiden eines Patienten. Geeignetere moderne statistische Auswerteverfahren (z.B. «mixed effect models») kamen zwar in den referierten Studien nicht zur Anwendung, aus dem Intent-to-treat-Modell oder den Completer- Analysen folgende Verzerrungen sind aber in der Mehrzahl der hier referierten Studien als eher gering zu betrachten, da der Prozentsatz von Dropouts generell niedrig lag. Zum Beispiel war ein Nebenergebnis der Frank-Studie, dass die Dropout-Rate bemerkenswert gering war, nämlich 17\% der 128 Patienten, die über einen Zeitraum von 3 Jahren der Langzeittherapie zugewiesen worden waren.

Zellgrößen: In vergleichenden Pharmako-Psychotherapiestudien überschreiten die Zellgrößen selten eine Anzahl von 20 Personen (Tab. 1). Diese Zellgrößen sind ausreichend um große Effekte zu entdecken, wie sie von Vergleichen zwischen Pharmakotherapie und Plazebo und zwischen Psychotherapie und Wartelisten-Kontrollgruppen erreicht werden. Aber die Zellgrößen sind zu klein um kleine bis mittlere Effektgrößen aufzudecken, wie sie aus dem Vergleich einer kombinierten Behandlung mit Einzelbehandlungsbedingungen resultieren dürften. Aus Studien die zwei aktive Behandlungsbedingungen miteinander vergleichen, weiß man, dass sich die Effektgrößen hier im Grenzbereich der klinischen Bedeutsamkeit bewegen, d.h. die Effekte sind von kleiner bis mittlerer Größe. Leider werden immer noch in der Mehrzahl der vergleichenden Behandlungsstudien keine Angaben über die Größe der Effekte gemacht und es ist daher schwer zu schätzen ob die Differenzen zwischen den Behandlungen klinisch bedeutsam sind und daher auch wert sind, weiter erforscht und entdeckt zu werden. Eine seltene Ausnahme bildet die Studie von Hollon et al. [1992], in der für den Vergleich zwischen kombinierter Behandlung und Pharmakotherapie eine Effektgröße von 0,44 und für den Vergleich von kombinierter Therapie und kognitiver Therapie eine Effektgröße von 0,35 berechnet worden ist. Um diese Effektgröße (z.B. m1-m2/SD
= 8) mit einer Wahrscheinlichkeit von $80 \%(\alpha=0,05)$ korrekt auf der HRSD zu entdecken ist bei zweiseitiger Fragestellung eine Zellgröße von mindestens 80 erforderlich. Daher war es in der Hollon-Studie schon aus statistischen Gründen nicht möglich einen statistisch signifikanten Unterschied zwischen der kombinierten Behandlung und Einzelbehandlung zu entdecken. Das trifft wahrscheinlich für die Mehrzahl zumindest der älteren Studien zu. Ausnahmen sind die großen neueren Studien z.B. von Paykel [Paykel et al., 1999] und Keller [Keller et al., 2000], die auch in der Tat signifikante Unterschiede zwischen der Kombinationsbehandlung und der Einzelbehandlung gefunden haben.

Mögliche Effekte durch Kombination zweier aktiver Behandlungsverfahren: Wenn man versucht die nicht signifikanten Ergebnisse zu erklären, die in vielen der Studien aufgetreten sind, gibt es mindestens zwei verschiedene mögliche Erklärungen [Beitman und Klerman, 1991], das «Größenmodell» und das «Häufigkeitsmodell»:

- Größenmodell: Dieses Modell geht davon aus, dass für jeden einzelnen Patienten eine Symptomverbesserung erreicht wird, die über dem Niveau liegt, das durch ein einzelnes Behandlungsverfahren erreicht wird. Ein solcher additiver Haupteffekt kann durch die üblichen varianzanalytischen Verfahren aufgedeckt werden. Diese Erklärung ist sozusagen identisch mit den oben genannten Effekt- und Zellgrößen und hat die Konsequenz, dass größere Stichproben notwendig sind um signifikante Unterschiede festzustellen.

- Häufigkeitsmodell: Das zweite Modell nimmt an, dass eine Symptomreduktion für die gesamte untersuchte Gruppe dadurch erreicht wird, dass die Wahrscheinlichkeit erhöht wird, dass ein Patient irgendeine Behandlung erhält, auf die er auch tatsächlich anspricht. Anders ausgedrückt: Die Nonresponder der einen Behandlung sind mögliche Responder der anderen Behandlung. In diesem Fall gibt es keinen wirklichen additiven Effekt, sondern eine beobachtete Symptomreduktion ist das Ergebnis einer Interaktion zwischen bereits vor der Intervention vorhandenen interindividuellen Unterschieden und der Art der Behandlung.

Für die Anwendung einer kombinierten Behandlung spräche streng genommen nur das Größenmodell, da im Falle des Häufigkeitsmodells die non-responder der einen Behandlung dann lediglich der anderen Behandlung zugewiesen werden müssten. Im letzteren Fall wäre also eine kombinierte Behandlung nicht notwendig. Das Zutreffen beider Modelle in Ihrer Reinform ist relativ unwahrscheinlich. Vielmehr spricht einiges für das Vorhandensein von Interaktionseffekten durch zumindest teilweise Überlappungen der unspezifischen und/oder spezifischen Behandlungskomponenten.

Interaktionseffekte zwischen Psychotherapie und Pharmakotherapie könnten eine nachweisbare geringe Überlegenheit der kombinierten Behandlung gegenüber der Einzelbehandlung prinzipiell erklären: Der Gesamteffekt der kombinierten Behandlung fällt durch solche Überlappungen geringer aus, 
als bei einer reinen Addition der Effekte von Psychotherapie und Pharmakotherapie. Leider kann zum jetzigen Zeitpunkt noch keine Aussage gemacht werden, wie groß die einzelnen spezifischen und unspezifischen Komponenten der Einzelbehandlungen im Vergleich zu der Kombinationsbehandlung sind. In den meisten hier referierten Studien liegen lediglich 3armige Designs vor, die keine Rückschlüsse auf die Art der Interaktion zulassen, da vor allem die unspezifischen Effekte in Form von Plazebo- und Aufmerksamkeitskontrollen meist nicht erfasst werden können.

Im Hinblick auf die hier dargestellten methodischen Gesichtspunkte lässt sich für künftige (Replikations-)Studien folgern, dass Studien mit mindestens 4-armigen Designs notwendig sind, um auf die Art der Interaktion zwischen Psychotherapie und Pharmakotherapie schließen zu können. Solange das nicht der Fall ist, wird man sich auf eine Erhöhung der Zellgrößen nach den oben gemachten (additiven) Annahmen stützen müssen.

\section{Diskussion}

In früheren Reviews wurde bereits geschlossen, dass in der Akutbehandlung einer MDD-Episode keine signifikante Überlegenheit einer kombinierten Psycho-Pharmakotherapie festgestellt werden kann, weder gegenüber Psychotherapie noch im Vergleich zur Medikation. In einigen der älteren kleineren Studien waren nicht-signifikante Trends berichtet worden, so dass man einen Nachweis der Signifikanz durch Erweiterung des Stichprobenumfangs oder durch Metaanalysen erwarten könnte. Die Stichprobenumfänge der neueren (Replikations-) Studien sind zwar größer als die der älteren, aber immer noch zu klein, um einen additiven Effekt nachweisen zu können. Die vorliegenden Metaanalysen konnten eine Überlegenheit der Kombinationsbehandlung gegenüber Psychotherapie und Pharmakotherapie nicht verifizieren.

Solange das Gegenteil nicht durch an größeren Stichproben durchgeführten Studien bewiesen ist, muss die folgende Schlussfolgerung gezogen werden: Die Ergebnisse der meisten systematischen Studien weisen darauf hin, dass es für die generelle Empfehlung einer kombinierten Pharmakotherapie und Psychotherapie keine ausreichende empirische Basis gibt. Bei der Langzeitbehandlung einer MDD ist eine kombinierte Behandlung gegenüber der Behandlung mit Psychotherapie alleine überlegen, nicht aber gegenüber der Behandlung mit Trizyklika [Frank et al., 1990]. Ergebnisse aus der Frank-Studie sind konsistent mit anderen kontrollierten Langzeitstudien zur Wirksamkeit von Imipramin [z.B. Keller, 1995] die zeigen, dass eine pharmakologische Langzeitbehandlung sehr effektiv sein kann, wenn die Medikation auf dem selben Dosierungsniveau gehalten wird wie zum Zeitpunkt des initialen Ansprechens. Selektive Serotonin-Wiederaufnahmehemmer rufen weniger unerwünschte Nebenwirkungen hervor und führen so zu einer geringeren Dropout-Rate [Lima und Moncrief, 2000]. In der Langzeitbehandlung älterer Patienten bringt eine Kombination von IPT und Medikation beachtliche Erfolge im Vergleich zu IPT alleine und als Trend auch im Vergleich zu Medikation alleine. Dieses Ergebnis steht im Widerspruch zu der verbreiteten Meinung, Psychotherapie sei nicht zur Behandlung älterer Patienten geeignet - sie scheinen im Gegenteil hier besonders zu profitieren.

In der Langzeitbehandlung ist die Compliance häufig ein groBes Problem, vor allem wenn man in Betracht zieht, dass ein Patient über eine Dauer von mehreren Jahren behandelt werden soll. Generell wird in den Studien zur Kombinationsbehandlung eine geringere Drop-out-Rate berichtet. Allerdings gibt es keine Unterschiede zwischen Kombinations- und Einzelbehandlung. Interessant war hier ein Ergebnis von Hautzinger et al. [1996]: Die Kombination von Pharmakotherapie mit Verhaltenstherapie erbrachte sogar ein erniedrigtes $\mathrm{Ni}$ veau der subjektiv empfundenen Nebenwirkungen im Vergleich zu der Bedingung Pharmakotherapie.

Am eindrucksvollsten konnte bisher der Vorteil einer kombinierten Behandlung, sowohl gegenüber Psychotherapie als auch gegenüber Pharmakotherapie, für die Subgruppe von Patienten mit chronisch verlaufender MDD gezeigt werden. Dabei handelt es sich um Patienten, die entweder zusätzlich zu einer Dysthymie an einer MDD (double depression), oder an einer anhaltenden MDD leiden [Keller et al., 2000], oder Patienten mit unvollständiger Remission zwischen den Episoden [Paykel et al., 1999].

Hingegen ist eine mittelschwere bis schwere akute Episode MDD eine klare Indikation für die Anwendung von Pharmakotherapie [Thase et al., 1997]. Psychotherapie alleine führt hier zu einer deutlich langsameren und insgesamt geringeren Symptomreduktion als ihre Kombination mit Antidepressiva.

\section{Dank}

Diese Arbeit wurde gefördert vom Bundesministerium für Bildung und Forschung (BMBF) im Rahmen des Förderschwerpunktes «Kompetenznetze in der Medizin». Wir danken außerdem folgenden Kolleginnen und Kollegen für Ihre Hilfe: Prof. Dr. M. Hautzinger, Dr. V. Henkel, Dipl.Psych. cand. S. Gratzmüller. 


\section{Literatur}

APA (American Psychiatric Association): Practice guidelines for major depressive disorder in adults. Am J Psychiatry 1993;150:1-26.

Blackburn IM, Bishop S, Glen AIM, Whalley LJ, Christie JE: The efficacy of cognitive therapy in depression: A treatment trial using cognitive therapy and pharmacotherapy, each alone and in combination. Br J Psychiatry 1981;139:181-189.

Beitman BD, Klerman GL (eds): Integrating Pharmacotherapy and Psychotherapy. Washington, American Psychiatric Press, 1991.

Conte HR, Plutchik R, Wild KV, Karasu TB: Combined psychotherapy and pharmacotherapy for depression. A systematic analysis of the evidence. Arch Gen Psychiatry 1986:43:471-479.

Covi L, Lipman RS: Cognitive behavioral group psychotherapy combined with imipramine in major depression. Psychopharmacol Bull 1987;23:173-176. deJong-Meyer R, Hautzinger M, Rudolf GA, Strauß W, Frick U: The effectiveness of antidepressants and cognitive-behavioral therapy, combined in patients with endogenous depression: Results of analyses of variance regarding main and secondary outcome criteria. Z Klin Psychol 1996;25:93-109.

Depression Guideline Panel: Depression in primary care: 2. Treatment of major depression (Clinical Practice Guideline No. 5, AHCPR Publication No. 93-0551). Rockville, U.S. Department of Health and Human Services, Public Health Service, Agency for Health Care Policy and Research, 1993.

Elkin I, Shea MT, Watkins JT, Imber SD, Stotsky SM, Collins JF, Glass DR, Pilkonis PA, Leber WR, Docherty JP, Fiester SJ, Parloff MB: National Institute of Mental Health Treatment of Depression Colaborative Research Program: General effectiveness of treatments. Arch Gen Psychiatry 1989;46:971-982.
Frank E, Kupfer DJ, Perel JM, Cornes C, Jarrett DB, Mallinger AG, Thase ME, McEachran AB, Grochocinski VJ: Three-year outcomes for maintenance therapies in recurrent depression. Arch Gen Psychiatry 1990;47:1093-1099.

Gaffan EA, Tsaousis I, Kemp WS: Researcher allegiance and meta-analysis: The case of cognitive therapy for depression. J Consult Clin Psychol 1995;63 966-980.

Hautzinger M, deJong-Meyer R, Treiber R, Rudolf GA, Thien U: Effectiveness of cognitive behavior therapy, pharmacotherapy, and the combination of both in nonendogenous unipolar depression. Z Klein Psychol 1996;25:130-145.

Hollon SD, Shelton RC, Loosen PT: Cognitive therapy and pharmacotherapy for Depression. J Consult Clin Psychol 1991;59:88-99.

Hollon SD, DeRubeis RJ, Evans MD, Wiemer MJ, Garvey MJ, Grove WM, Tuason VB: Cognitive therapy and pharmacotherapy for depression singly and in combination. Arch Gen Psychiatry 1992;49:774-81.

Keller MB, Harrison W, Fawcett JA, Gelenberg A, Hirschfeld RM, Klein D, Kocsis JH, McCullough JP, Rush AJ, Schatzberg A, Russell J, Hirschfeld R, Klein D, McCullough JP, Fawcett JA, Kornstein S, LaVange L, Harrison W: Treatment of chronic depression with sertraline or imipramine: Preliminary blinded response rates and high rates of undertreatment in the community. Psychopharmacol Bull 1995;31:205-212.

Keller MB, McCullough JP, Klein DN, Arnow B, Dunner DL, Gelenberg AJ, Markowitz JC, Nemeroff CB, Russell JM, Thase ME, Trivedi MH, Zajecka J: A comparison of nefazodone, the cognitive behavioral-analyis system of psychotherapy, and their combination for the treatment of chronic. N Engl J Med 2000;342: 1462-1470.

Kupfer DJ: Lessons to be learned from long-term treatment of affective disorders: Potential utility in panic disorder. J Clin Psychiatry 1991;52(Suppl 2): $12-16$.
Lima MS, Moncrieff JA: Comparison of drugs versus placebo for the treatment of dysthymia (Cochrane Review), http://www.update-software.com/abstracts/ab 001130.htm, 2000

Murphy GE, Simons AD, Wetzel RD, Lustman PJ: Cognitive therapy and pharmacotherapy. Singly and together in the treatment of depression. Arch Gen Psychiatry 1984;41:33-41.

Mynors-Wallis WL, Gath DH, Day A, Baker F: Randomised controlled trial of problem solving treatment, antidepressant medication, and combined treatment for major depression in primary care. BMJ 2000;320: $26-30$.

Paykel ES, Scott J, Teasdale JD, Johnson AL, Garland A, Moore R, Jenaway A, Cornwall PL, Hayhurst H, Abbott R, Pope M: Prevention of relapse in residual depression by cognitive therapy. A controlled trial. Arch Gen Psychiatry 1999;56:829-835.

Reynolds CF 3rd, Frank E, Perel JM, Imber SD, Cornes C, Miller MD, Mazumdar S, Houck-PR, Dew MA, Stack JA, Pollock BG, Kupfer DJ: Nortriptyline and interpersonal psychotherapy as maintenance therapies for recurrent major depression: A randomized controlled trial in patients older than 59 years. JAMA 1999;281:39-45.

Robinson LA, Berman JS, Neimeyer RA: Psychotherapy for the treatment of depression: a comprehensive review of controlled outcome research. Psychol Bull 1990;108:30-49.

Sackett DL: Evidence-based medicine. Spine 1998; 15,23,10:1085-1086

Thase ME, Greenhouse JB, Frank E, Reynolds CFI, Pilkonis PA, Hurley K, Grochocinski V, Kupfer DJ: Treatment of major depression with psychotherapy or psychotherapy-pharmacotherapy combinations. Arch Gen Psychiatry 1997;54:1009-1015. 